\title{
Attitude Control of a Quadcopter Platform Based on Fractional Control Laws
}

\author{
Taha Ennaciri ${ }^{*}$, Nabil Mrani ${ }^{2}$, Ahmed El abbassi ${ }^{3}$ and Jaouad Foshi ${ }^{4}$ \\ 134 Team Electronics, Instrumentation and Measurement Physics, FST Errachidia \\ Moulay Ismaïl University - Morocco \\ ${ }^{2}$ Team Technologie d'Information et du Multimedia (TIM) \\ EST Meknès, Moulay Ismaïl University - Morocco \\ ${ }^{1}$ taha.nasr14@gmail.com ${ }^{2}$ nmrani@gmail.com, ${ }^{3}$ a.elabbassi@fste.umi.ac.ma, ${ }^{4}$ j.foshi@fste.umi.ac.ma
}

\begin{abstract}
The purpose of this study, is to establish a comparison between two types of controllers, to be used to control the attitude of an unmanned aerial vehicle UAV, known as quadcopter platform. The two types of controllers, assumed in this work, are: A classic Linear Quadratic Controller LQR, and Controller based on fractional control laws. The proposed method shows its advantages in terms of time response and the settling down to the desired attitude, compared the LQR controller.
\end{abstract}

Keywords: Fractional Control, LQR, UAV.

\section{Introduction}

Control methods has been improved and developed in the last decades. This leads to an increase of the use of several modern technologies, such as the Unmanned Aerial Vehicles (UAV) [1].

Lately, many methodologies see the light of the day, such as fractional control, optimal control, linear quadratic control [2], etc. Different sensors and actuators have been also used, such as attitude control with tube, using Magnetorquers, using Reaction wheels etc.

In this work, we will elaborate both linear quadratic control and fractional controllers for UAV attitude stabilization, in order to compare them. Firstly we will describe the dynamics of spacecraft. Next, we will introduce and discuss Linear Quadratic Control and fractional control law for the quadcopter platform attitude. This work aims at obtaining comparative results between the two methods.

\section{Quadcopter attitude dynamic model}

The vertical movement represents the quadrotor changing its vertical speed and position. This movement is described by the following equations [3, [4] :

$$
\begin{aligned}
& \dot{z}=w, \\
& \dot{w}=-2 \frac{b}{m} \Omega_{0}\left(\Omega_{1}+\Omega_{3}-\Omega_{2}+\Omega_{4}\right), \\
& \dot{\Omega}_{i}=-10 \Omega_{i}+7 U_{i} \quad i=\{1,2,3,4\} .
\end{aligned}
$$

The lateral movement is achieved when the quadrotor is keeping its vertical position $z$, longitudinal position $x$ and its yaw angle. This type of movement is described by the equations [3], [4]:

${ }^{*}$ Corresponding author.Taha Ennaciri ${ }^{1}$ taha.nasr14@gmail.com 2010 Mathematics Subject Classification: 26A33. 


$$
\begin{aligned}
& \dot{y}=v, \\
& \dot{\phi}=p, \\
& \dot{v}=g \phi, \\
& \dot{p}=2 \frac{l b}{I_{x}} \Omega_{0}\left(\Omega_{2}-\Omega_{4}\right), \\
& \dot{\Omega}_{i}=-10 \Omega_{i}+7 U_{i} \quad i=\{2,4\} .
\end{aligned}
$$

The longitudinal movement is represented by the quadrotor keeping its vertical and lateral position and maintaining its yaw angle. This type of movement is described by the equations [3], 4]:

$$
\begin{aligned}
& \dot{x}=u, \\
& \dot{\theta}=q, \\
& \dot{u}=-g \theta, \\
& \dot{q}=2 \frac{l b}{I_{y}} \Omega_{0}\left(\Omega_{1}-\Omega_{3}\right), \\
& \dot{\Omega}_{i}=-10 \Omega_{i}+7 U_{i} \quad i=\{1,3\} .
\end{aligned}
$$

The yawing movement is the movement when the quadrotor is keeping its position and only its yaw angle changes. This movement is described by the following equations [3], 4]:

$$
\begin{aligned}
& \dot{\psi}=r, \\
& \dot{r}=2 \frac{d}{I_{Z}} \Omega_{0}\left(\Omega_{1}+\Omega_{2}+\Omega_{3}+\Omega_{4}\right), \\
& \dot{\Omega}_{i}=-10 \Omega_{i}+7 U_{i} \quad i=\{1,2,3,4\}
\end{aligned}
$$

In the above equations, we denote by:

- $\Omega_{0}$ is the angular rate of the propeller during the hover,

- $b$ is the thrust constant,

- $d$ Drag factor of the rotating propeller,

- $z$ is the vertical coordinate in Earth-fixed frame,

- $\omega$ is the vertical speed in body-fixed frame,

- $\Omega_{n}$ is the angular rate of each propeller,

- $m$ is the Mass of the quadrotor,

- $I_{R}, I_{T}$ and $I_{L}$ the moments of inertia corresponding respectively to roll, pitch and yaw axis,

- $\phi, \theta$ and $\psi$ are roll, pitch and yaw assumed small in the attitude control,

- $p$ Roll rate,

- $q$ Pitch rate,

- $r$ Yaw rate,

- $u$ Longitudinal speed in body-fixed frame,

- $v$ Lateral speed in body-fixed frame,

- $w$ Vertical speed in body-fixed frame,

- $x$ Longitudinal coordinate in Earth-fixed frame,

- $y$ Lateral coordinate in Earth-fixed frame,

- $z$ Vertical coordinate in Earth-fixed frame. 
The state space representation or the state model of the systems (1), (2), (3) and (4) is written as below:

$$
\left\{\begin{array}{l}
\dot{x}=A x(t)+B u(t) \\
y=C x(t)+D u(t) .
\end{array}\right.
$$

In the above equations, we denote by:

$$
\begin{gathered}
A=\left(\begin{array}{cccccc}
0 & 1 & 0 & 0 & 0 & 0 \\
0 & 0 & -\frac{b \Omega_{0}}{m} & \frac{b \Omega_{0}}{m} & -\frac{b \Omega_{0}}{m} & \frac{b \Omega_{0}}{m} \\
0 & 0 & -10 & 0 & 0 & 0 \\
0 & 0 & 0 & -10 & 0 & 0 \\
0 & 0 & 0 & 0 & -10 & 0 \\
0 & 0 & 0 & 0 & 0 & -10
\end{array}\right) ; B=\left(\begin{array}{c}
0 \\
0 \\
1 \\
-1 \\
1 \\
-1
\end{array}\right) \\
C=\left(\begin{array}{lllllll}
1 & 0 & 0 & 0 & 0 & 0 & 0
\end{array}\right) ; x=\left(\begin{array}{c}
z \\
\omega \\
\Omega_{1} \\
\Omega_{2} \\
\Omega_{3} \\
\Omega_{4}
\end{array}\right) \\
D=0 .
\end{gathered}
$$

For system (2):

$$
\begin{gathered}
A=\left(\begin{array}{cccccc}
0 & 0 & 1 & 0 & 0 & 0 \\
0 & 0 & 0 & 1 & 0 & 0 \\
0 & -g & 0 & 0 & 0 & 0 \\
0 & 0 & 0 & 0 & 2 \frac{l b \Omega_{0}}{I_{y}} & -2 \frac{l b \Omega_{0}}{I_{y}} \\
0 & 0 & 0 & 0 & -10 & 0 \\
0 & 0 & 0 & 0 & 0 & -10
\end{array}\right) ; B=\left(\begin{array}{c}
0 \\
0 \\
0 \\
0 \\
1 \\
-1
\end{array}\right) \\
C=\left(\begin{array}{ccccccc}
0 & 1 & 0 & 0 & 0 & 0 & 0
\end{array}\right) ; x=\left(\begin{array}{c}
y \\
v \\
p \\
\Omega_{3} \\
\Omega_{4}
\end{array}\right) \\
D=0 .
\end{gathered}
$$

For system (3):

$$
\begin{gathered}
A=\left(\begin{array}{cccccc}
0 & 0 & 1 & 0 & 0 & 0 \\
0 & 0 & 0 & 1 & 0 & 0 \\
0 & g & 0 & 0 & 0 & 0 \\
0 & 0 & 0 & 0 & 2 \frac{l b \Omega_{0}}{I_{x}} & -2 \frac{l b \Omega_{0}}{I_{x}} \\
0 & 0 & 0 & 0 & -10 & 0 \\
0 & 0 & 0 & 0 & 0 & -10
\end{array}\right) ; B=\left(\begin{array}{c}
x \\
\theta \\
0 \\
0 \\
0 \\
1 \\
-1
\end{array}\right) \\
C=\left(\begin{array}{llllllc}
0 & 1 & 0 & 0 & 0 & 0 & 0
\end{array}\right) ; x=\left(\begin{array}{c}
0 \\
u \\
q \\
\Omega_{1} \\
\Omega_{2}
\end{array}\right)
\end{gathered}
$$




$$
D=0
$$

For system (4):

$$
\begin{gathered}
A=\left(\begin{array}{cccccc}
0 & 1 & 0 & 0 & 0 & 0 \\
0 & 0 & 2 \frac{d \Omega_{0}}{I_{z}} & 2 \frac{d \Omega_{0}}{I_{z}} & 2 \frac{d \Omega_{0}}{I_{z}} & 2 \frac{d \Omega_{0}}{I_{z}} \\
0 & 0 & -10 & 0 & 0 & 0 \\
0 & 0 & 0 & -10 & 0 & 0 \\
0 & 0 & 0 & 0 & -10 & 0 \\
0 & 0 & 0 & 0 & 0 & -10
\end{array}\right) ; B=\left(\begin{array}{c}
0 \\
0 \\
1 \\
-1 \\
1 \\
-1
\end{array}\right) \\
C=\left(\begin{array}{lllllll}
1 & 0 & 0 & 0 & 0 & 0 & 0
\end{array}\right) ; x=\left(\begin{array}{c}
\psi \\
r \\
\Omega_{1} \\
\Omega_{2} \\
\Omega_{3} \\
\Omega_{4}
\end{array}\right) \\
D=0 .
\end{gathered}
$$

\section{Control laws}

\subsection{Linear quadratic control}

In order to design a controller that is based on Linear Quadratic Regulator, several conditions and constraints must be satisfied.

For a given controlled process described by the state equation below:

$$
\dot{x}=A x(t)+B u(t)
$$

To design a LQR controller we must:

- Find an admissible control law which minimizes the cost criterion given below:

$$
J=\int_{0}^{\infty}\left(x^{T}(t) Q x(t)+u^{T}(t) R u(t)\right) d t,
$$

where $Q$ is a positive semi definite matrix and $R$ is a positive definite matrix.

- Find a unique solution $P$, in the class of positive semidefinite matrices, to the algebraic Riccati equation, in order to find the gain $K$ which determinate the control law $u$.

We have successively: - Algebraic Riccati Equation :

$$
A^{T} P+P A-P B R^{-1} B^{T} P+Q=0
$$

- The Gain $K$ :

$$
K=R^{-1} B^{T} P
$$

- Control law:

$$
u(t)=-K x(t)
$$

For the uniqueness of the solution $P,(A, B)$ must be controllable, and $(Q, A)$ must be observable, which is has been verified for all systems.

$$
R=1 ; Q=\operatorname{diag}\left(\begin{array}{llllll}
10^{7} & 1 & 1 & 1 & 1 & 1
\end{array}\right)
$$




\section{$3.2 \quad$ Fractional control}

In order to establish a fractional controller, the control law for all systems must be written as:

$$
u(t)=-k x^{(\alpha)}(t)
$$

where $\alpha$ is a real number representing the order of derivation of $x(t)$.

The equation (6) becomes:

$$
\dot{x}(t)=A x(t)-B K x^{(\alpha)}(t) .
$$

The definition given by Grünwald-Letnikov [5] for a fractional derivative using the Gamma function is:

$$
x^{(\alpha)}(t)=\lim _{h \rightarrow 0} \frac{1}{h^{(\alpha)}} \sum_{k=0}^{\infty}(-1)\left(\begin{array}{c}
\alpha \\
k
\end{array}\right) x(t-k h)
$$

where

$$
\left(\begin{array}{l}
\alpha \\
k
\end{array}\right)=\frac{\Gamma(\alpha+1)}{\Gamma(k+1) \Gamma(\alpha-k+1)} .
$$

\section{Simulations and results}

For all the simulations made in this paper we consider the following parameters:

$I_{x}=0.0093 \mathrm{~m}^{2} \mathrm{~kg}$

$I_{y}=0.0092 \mathrm{~m}^{2} \mathrm{~kg}$

$m=1.043 \mathrm{~kg}$

$l=0.275 \mathrm{~m}$

$I_{z}=0.0151 \mathrm{~m}^{2} \mathrm{~kg}$

$b=1.5108 \times 10^{-5} \mathrm{~kg} . \mathrm{m}$

$\Omega_{0}=411.4743 \frac{\mathrm{rad}}{\mathrm{s}}$

Note that the gain matrix $K$ is separately calculated for all four systems.

When $\alpha=0: u(t)=-k x^{(\alpha)}(t)=-k x(t)$. This corresponds to an LQR controller.

Figure (1) illustrates the step response for different values of $\alpha$ between -0.2 and 0.2 , within a step of 0.1 .

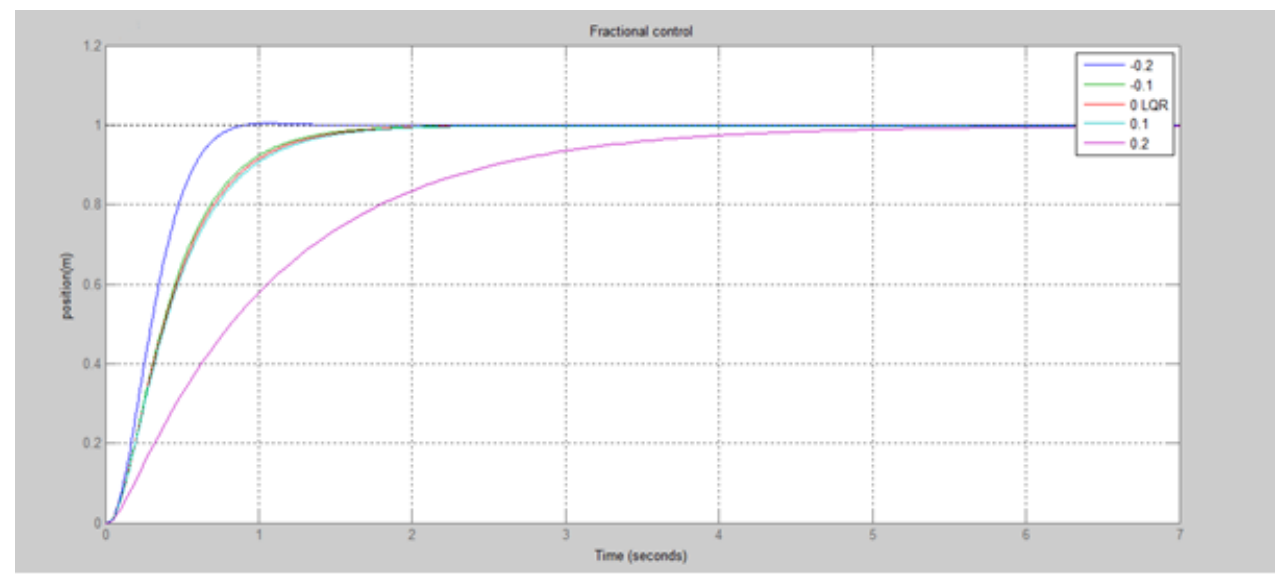

Fig. 1: Vertical position step response

It is noticed that, the step response for the vertical position of the plant, have no overshooting value and have an accommodating time of two seconds for the LQR control. However the fractional control, shows a quick accommodating time for the negative values of $\alpha$.

Figures (2), (3) and (4) illustrate the evolution of the Roll, Pitch and Yaw angles, respectively, versus time for different values of $\alpha$ between -0.2 and 0.2 , within a step of 0.1 . 


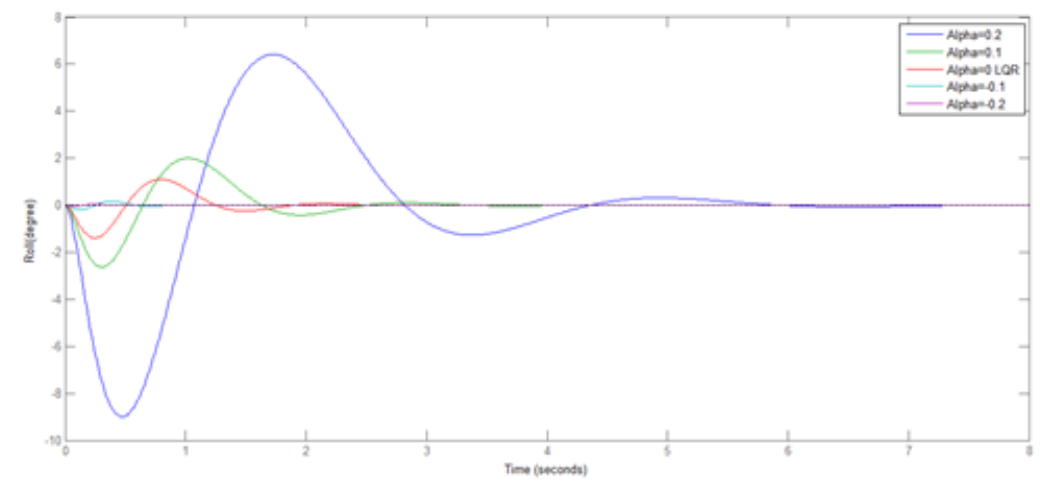

Fig. 2: Evolution of the Roll angle versus time

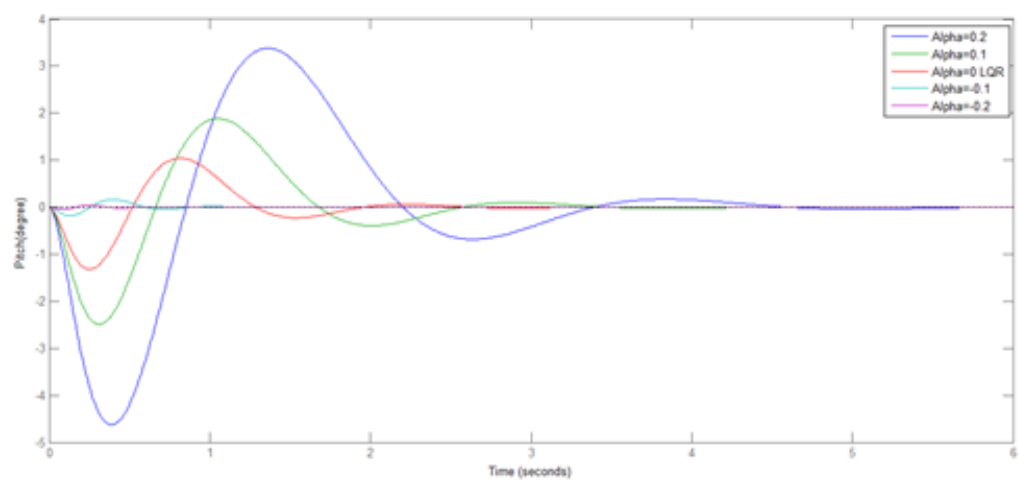

Fig. 3: Evolution of the Pitch angle versus time

It is noticed that for negative values of $\alpha$, the system is settling quickly, and has better precision compared to a LQR controller.

Taking for example: $\alpha=-0.2$; Roll angle for the Fractional control:

- The settling time is : 0.6 second

For the LQR control (Roll angle):

- The settling time is : 2.8 second

In this case the fractional controller, shows better performance in terms of rapidity, compared to the LQR one. Some fluctuations are noted for certain values of $\alpha$, considering the Roll and Pitch angles, the quadcopter will experience some ups and downs before it settles to zeros. 


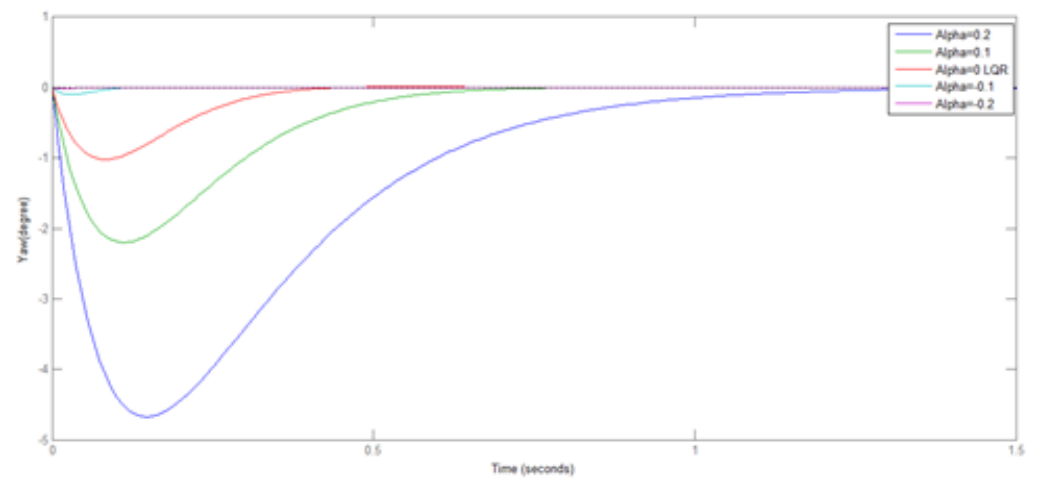

Fig. 4: Evolution of the Yaw angle versus time

\section{Conclusion}

Considering the previous analysis and results found in this work, it is easy to observe that, the plant was controlled with two different control methods, presenting satisfactory but different results.

It is known that, the LQR controllers are robust, and produce a very low steady state error, but with a big transition delay and slow settling time. With the new controller -Fractional control- we manage to take the advantages of the LQR one, with short transition delay and a faster settling time.

\section{References}

[1] S. Gupte, P. Mohandas and J. M. Conrad. A survey of quadrotor unmanned aerial vehicle, Proceedings of the IEEE SoutheastCon, Orlando, Florida, USA, (2012), pp. 1-6.

[2] A. Kailil, N. Mrani, M. Mliha Touati, S. Choukri and N. Elalami. Low Earth-orbit satellite attitude stabilization with fractional regulators, International Journal of Systems Science, Vol. 35, No. 10, (2004), pp. 559-568.

[3] A. L. Salih, M. Moghavvemi, H. A. F. Mohamed and K. S. Gaeid. Flight PID controller design for a UAV quadrotor, Scientific Research and Essays, Vol. 5, (2010), pp. 3360-3367.

[4] B. D. O. Anderson and J. B. Moore. Optimal Control - Linear Quadratic Methods, Prentice Hall, (1989).

[5] Ivo Petráš. Fractional Derivatives, Fractional Integrals, and Fractional Differential Equations in Matlab, Engineering Education and Research Using MATLAB, InTech, (2011), pp. 239-264. 